\title{
Interface controlled electronic variations in correlated heterostructures
}

\author{
K. Gehrke, V. Moshnyaga, and K. Samwer \\ I. Physikalisches Institut, Georg-August-Universität Göttingen, Friedrich-Hund-Platz 1, 37077 Göttingen, Germany \\ O. I. Lebedev, J. Verbeeck, D. Kirilenko, and G. Van Tendeloo \\ EMAT, University of Antwerp, Groenenborgerlaan 171, B-2020 Antwerpen, Belgium \\ (Received 9 December 2009; revised manuscript received 15 May 2010; published 7 September 2010)
}

\begin{abstract}
An interface modification of $(\mathrm{LaCa}) \mathrm{MnO}_{3}-\mathrm{BaTiO}_{3}$ superlattices was found to massively influence magnetic and magnetotransport properties. Moreover it determines the crystal structure of the manganite layers, changing it from orthorhombic (Pnma) for the conventional superlattice (cSL), to rhombohedral $(R \overline{3} c)$ for the modified one (mSL). While the cSL shows extremely nonlinear ac transport, the $\mathrm{mSL}$ is an electrically homogeneous material. The observations go beyond an oversimplified picture of dead interface layers and evidence the importance of electronic correlations at perovskite interfaces.
\end{abstract}

DOI: 10.1103/PhysRevB.82.113101

PACS number(s): 75.47.Lx, 71.38.Mx, 73.20.Mf, 73.21.Cd

Interfaces of complex oxides have gained much attention since the discovery of a high-mobility quasi-twodimensional electron gas at the $\mathrm{TiO}_{2} / \mathrm{LaO}$ interface between insulating $\mathrm{LaAlO}_{3}$ (LAO) and $\mathrm{SrTiO}_{3}$ (STO). ${ }^{1,2}$ This unexpected finding disclosed a new role of interfaces in oxide heterostructures. Thereby the reconstruction of the interface is discussed to avoid an electrostatic potential, otherwise building up in the lanthanum perovskite. For this reconstruction either electrons redistribute or ions rearrange through relaxation or deviation from stoichiometry. The charge transfer can effectively dope the materials in a rather thin region in the vicinity of the interface $e^{3,4}$ and the resulting change in carrier concentration due to the presence of an interface can be termed "interface doping." For strongly correlated electron systems it is well known that the carrier concentration massively influences the properties of the material. A prominent example is the class of perovskite manganites, which are interesting not only because of high spin polarization in the ferromagnetic phase, ${ }^{5,6}$ but also due to the fascinating rich magnetic phase diagram that opens up upon doping. ${ }^{7}$ The ground state of $\mathrm{La}_{1-x} \mathrm{Ca}_{x} \mathrm{MnO}_{3}$ changes from ferromagnetic (FM) metallic for $0.2 \leq x \leq 0.4$ to antiferromagnetic (AFM) insulating for $x \geq 0.5$, whereas the phase boundary is not a sharp line, but rather a broad region around $x=0.45$ where FM and AFM phases coexist. ${ }^{8}$ The FM state is stabilized by gaining kinetic energy due to the delocalization of charge carriers at the expense of antiferromagnetic exchange of localized spins. Localization is stabilized by the JahnTeller (JT) effect, that lifts the $e_{\mathrm{g}}$-orbital degeneracy of $\mathrm{Mn}^{3+}$ ions, ${ }^{9}$ giving rise to JT polarons, that are discussed to be the main type of charge carrier at all temperatures. ${ }^{10}$ Binding of these JT polarons into pairs of correlated polarons or bipolarons is now argued to bring about the strong localization at the metal-insulator (MI) transition. ${ }^{11}$ Even for optimaldoping $(0.2 \leq x \leq 0.4)$ correlated JT polarons have been observed by neutron and $\mathrm{x}$-ray scattering ${ }^{12,13}$ with the wave vector of these short-range JT-distorted regions being $\vec{q}=\left[\frac{1}{4}, \frac{1}{4}, 0\right]$.

The loss of FM order in $\mathrm{La}_{2 / 3} \mathrm{Ca}_{1 / 3} \mathrm{MnO}_{3}$ (LCMO) and $\mathrm{La}_{2 / 3} \mathrm{Sr}_{1 / 3} \mathrm{MnO}_{3}$ (LSMO) below a critical film thickness, ${ }^{14,15}$ and the poor performance of LSMO-STO-LSMO tunnel junctions at elevated temperatures, ${ }^{16,17}$ have been connected to the weakening of double exchange at manganite interfaces. The breakup of the $\mathrm{Mn}-\mathrm{O}$ chains has been accounted for this interface-induced phase separation ${ }^{18}$ and the carrier depletion (interface doping), alike the one discussed for LAO/STO interfaces, seems to be a driving force of the localization. Moreover it has been shown that two monolayers of $\mathrm{LaMnO}_{3}$ (LMO) introduce extra carriers to the interface region, counteracting the depletion and stabilizing the FM/ spin polarization. ${ }^{16,17,19}$ Elastic constraints also play an important role since lattice relaxations are intrinsically coupled to the electronic correlations in Jahn-Teller systems. ${ }^{20}$ Furthermore $\mathrm{CP}$ can be attributed to the CE-AFM phase, which shows the same superstructure wave vector $\vec{q}=\left[\frac{1}{4}, \frac{1}{4}, 0\right]$. As we have shown recently, $\mathrm{CP}$ can be probed by means of the third harmonic voltage, which is a measure of electric nonlinearity, because of their quadrupole nature. ${ }^{21}$ Here we use ac transport, superconducting quantum interference device magnetometry and transmission electron microscopy (TEM) to study the influence of interfaces in superlattices (SLs) and the effect of an interface modification by additional LMO layers.

We compare the physical properties of two manganitetitanate SLs. The conventional SL (cSL) consists of 40 unit cells (u.c.) LCMO and 20 u.c. $\mathrm{BaTiO}_{3}$ (BTO), repeated ten times $\left[\mathrm{LCMO}_{40} / \mathrm{BTO}_{20}\right]_{10}$. The modified SL (mSL) consists of the same LCMO and BTO layers, but additionally two u.c. of $\mathrm{LMO}$ were introduced at each interface: $\left[\mathrm{LCMO}_{40} / \mathrm{LMO}_{2} / \mathrm{BTO}_{20} / \mathrm{LMO}_{2}\right]_{10}$. The nominal thickness of the additional LMO layers has been controlled by the volume of metal-organic solution introduced to the deposition system. The SLs were grown on MgO (100) substrates at $T=900{ }^{\circ} \mathrm{C}$ by means of a metal-organic aerosol deposition technique. ${ }^{22}$ The monolayer accuracy during deposition was achieved by controlling the volume of the metal-organic solution for each layer. The termination of the individual layers however cannot currently be controlled by this method.

The x-ray diffraction (XRD) $(\Theta-2 \Theta)$ patterns show typical satellite peaks, arising from the SL periodicity (Fig. 1). The calculated periodicities from Schuller's formula ${ }^{23}$ are $\lambda_{\mathrm{cSL}}=23.3 \pm 0.05 \mathrm{~nm}$ and $\lambda_{\mathrm{mSL}}=25.3 \pm 0.05 \mathrm{~nm}$, which is 


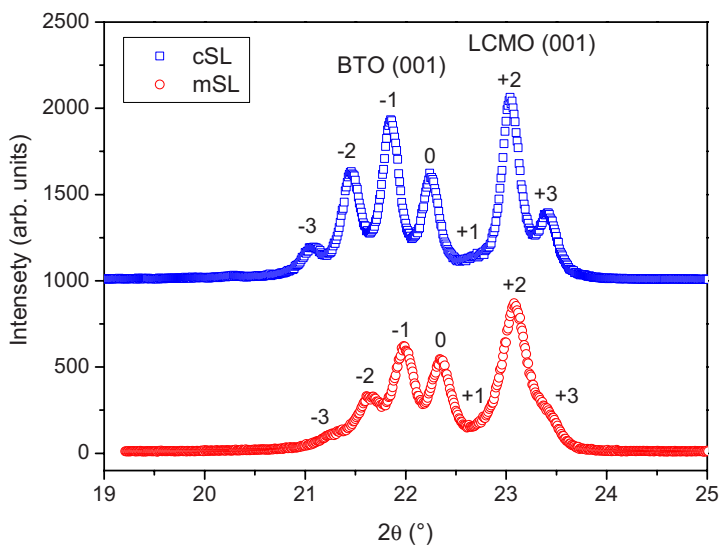

FIG. 1. (Color online) X-ray diffraction patterns of the conventional (open squares) and modified (open circles) superlattice at the position of the perovskite (001) Bragg peak. Overall intensity is modulated by the BTO (001) and LCMO (001) crystalline reflexes.

in a good agreement with the nominal thicknesses. Rocking curves obtained by XRD show very broad distributions for both SLs $\left(\mathrm{FWHM}_{\mathrm{cSL}}=1.18^{\circ} \pm 0.01^{\circ}\right.$ and $\mathrm{FWHM}_{\mathrm{mSL}}$ $=1.38^{\circ} \pm 0.01^{\circ}$ ) which we attribute partly to the low quality of $\mathrm{MgO}$ substrates. A detailed study of the crystal and chemical structure of SLs was performed on cross section and plan-view specimens by means of TEM. Plan-view and cross-section electron-diffraction (ED) [Figs. 2(a) and 2(b)] patterns clearly show heteroepitaxial growth of all layers for both SLs. Cross-section high-resolution TEM (HRTEM) images confirm epitaxial growth of SLs and show coherent, atomically flat, and sharp interfaces [Figs. 1(c) and 1(d)]. The extra LMO layers in $\mathrm{mSL}$ are not visible in HRTEM image but, obviously, introduce significant structural differences between cSL and mSL as it can be detected by ED and HRTEM. The LCMO layer of the mSL exhibits the unusual $R \overline{3} c$ structure in contrast to most bulk samples ${ }^{24}$ and cSL LCMO showing both Pnma structure. The lattice parameters of the manganite layers in pseudocubic notation are $a_{\mathrm{cSL}}=3.87 \pm 0.02 \AA$ and $c_{\mathrm{cSL}}=3.82 \pm 0.02 \AA$ for the conventional superlattice and $a_{\mathrm{mSL}}=3.88 \pm 0.01 \AA$ and $c_{\mathrm{mSL}}=3.87 \pm 0.01 \AA$ for the modified superlattice. The structure of BTO of both SLs is found to be close to $P 4 \mathrm{~mm}$. The tetragonality of the BTO layers is $\frac{c}{a}=1.04 \pm 0.005$ for the conventional and $\frac{c}{a}=1.025 \pm 0.005$ for the modified SL. Energy-filtered TEM of mSL (Fig. 3) shows well-defined chemical separation of LCMO and BTO layers. Intermixing of LCMO and LMO on the small scale of several monolayers nonetheless can not be excluded. In order to analyze strain fields, the geometrical phase analysis (GPA) method ${ }^{25}$ has been applied to HRTEM images of cSL and mSL. The results of GPA along the growth direction are shown in Figs. 2(e) and 2(f) and reveal inhomogeneously strained cSL, in contrast to the mSL heterostructure which looks homogeneously strained.

Magnetic properties of the SLs have been measured with external field applied parallel to the film plane. The temperature dependence of the magnetization of both SLs, see Fig. 4(a) shows a rather broad ferromagnetic transition, typical for thin LCMO layers. ${ }^{26,27}$ The magnetic Curie temperatures

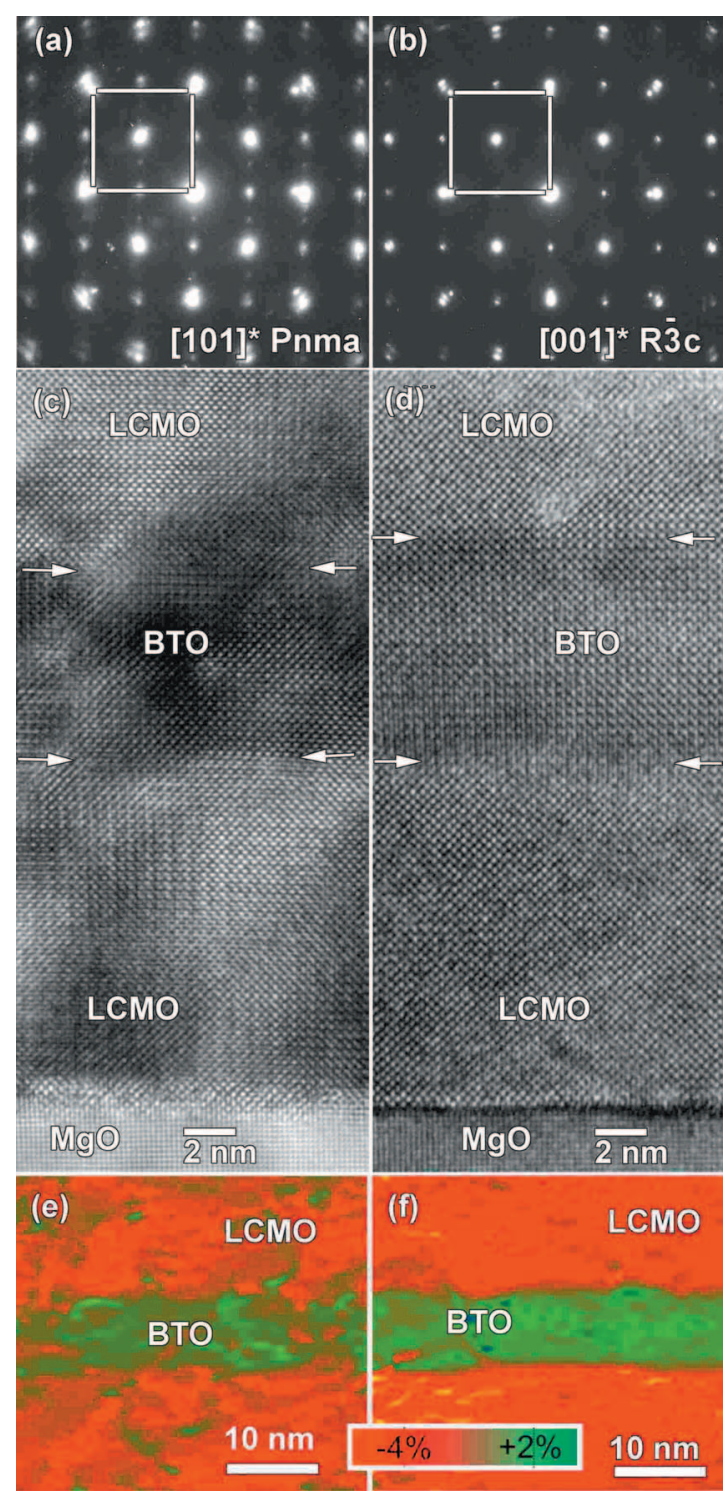

FIG. 2. (Color) Microstructure analysis of the cSL (left) and mSL (right). Electron-diffraction patterns (top) reveal (a) Pnma symmetry for cSL and (b) $R \overline{3} c$ symmetry for mSL. Cross-section HRTEM (mid) confirm epitaxial growth and coherent interfaces of (c) cSL and (d) mSL. GPA (bottom) shows strain fields along the growth direction to be (e) inhomogeneous for cSL and (f) homogeneous for $\mathrm{mSL}$.

of the SLs are $T_{C}^{\mathrm{cSL}}=245 \mathrm{~K}$ and $T_{C}^{\mathrm{mSL}}=253 \mathrm{~K}$. Magnetic hysteresis $M(H)$, shown in Fig. 4(b) was measured at $T=10 \mathrm{~K}$ after cooling without [zero-field cooling (ZFC)] and with an external field $\mu_{0} H=5 \mathrm{~T}$ applied during cooling (FC). The saturation magnetization $\left(M_{\mathrm{s}}\right)$ of the SLs after FC is as large as $M_{s}^{\mathrm{cSL}}=287 \mathrm{emu} \mathrm{cm}^{-3}$ and $M_{s}^{\mathrm{mSL}}=393 \mathrm{emu} \mathrm{cm}{ }^{-3}$, which is smaller than the bulk value, $M_{\mathrm{s}}^{\text {bulk }} \approx 650 \mathrm{emu} \mathrm{cm}^{-3}$. In the case of the $\mathrm{mSL}$, additional LMO layers are expected to contribute to the magnetic moment and therefore the volumes of both LCMO and LMO were considered calculating $M_{s}^{\mathrm{mSL}}$. The corresponding coercivities are $H_{c}^{\mathrm{cSL}}=721 \mathrm{Oe}$ and $H_{c}^{\mathrm{mSL}}=536$ Oe. Interestingly, after ZFC the $M_{s}$ of both SLs were smaller than $M_{s}$ after FC, $\frac{\Delta M_{s}}{M_{s}} \approx-11 \%$ for the cSL and $\frac{\Delta M_{s}}{M_{s}} \approx-3 \%$ for the mSL. 

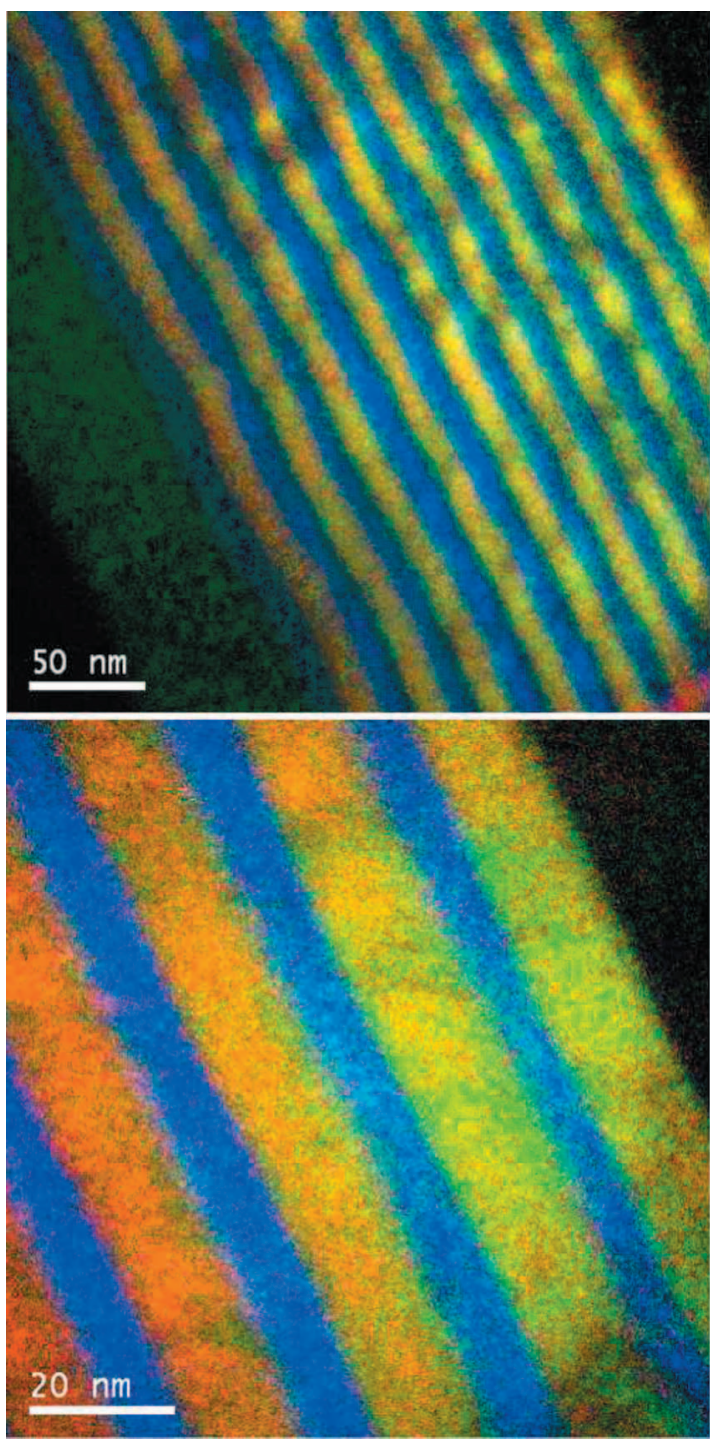

FIG. 3. (Color) Energy-filtered transmission electron micrographs of the modified superlattice (blue $=\mathrm{Ti}$, red $=\mathrm{Mn}$, and green $=\mathrm{La}$ ). Film plus substrate (top) and topmost layers of the film (bottom).

Measurements of the ac electric transport were performed by means of four-probe method with silver paste contacts at the edges of the SLs. The ac-current amplitude was $I_{\mathrm{ac}}=10 \mu \mathrm{A}$ and the frequency $f_{\mathrm{ac}}=17 \mathrm{~Hz}$. Besides the linear electric response with resistance $R_{\omega}$ at the fundamental frequency $f_{\text {ac }}$, we report the third harmonic response at $f_{3 \omega}=3 f_{\text {ac }}$ in terms of the coefficient $K_{3 \omega}=\frac{U_{3 \omega}}{U_{\omega}}$, which is a measure of the electrical nonlinearity in the film. ${ }^{21}$ Figure 5(a) shows the temperature dependence of $R_{\omega}$, measured after FC and ZFC. The cSL shows a large difference (factor of 10) between FC and $\mathrm{ZFC}$ resistance at low temperature, $T=10 \mathrm{~K}$. The difference gets smaller at $T \approx 150 \mathrm{~K}$ and vanishes completely close to $T_{c}=245 \mathrm{~K}$. The MI transition of the cSL is shifted by $\Delta T=77 \mathrm{~K}$ below the magnetic transition. For the mSL no difference between $R_{\omega}^{\mathrm{mSL}}(\mathrm{FC})$ and $R_{\omega}^{\mathrm{mSL}}(\mathrm{ZFC})$ was observed, and $T_{c}^{\mathrm{mSL}}-T_{\mathrm{MI}}^{\mathrm{mSL}}=22 \mathrm{~K}$. Regarding nonlinear transport, shown in Fig. 5(b), the cSL shows a strong increase in $K_{3 \omega}$ by decreasing temperature, both for
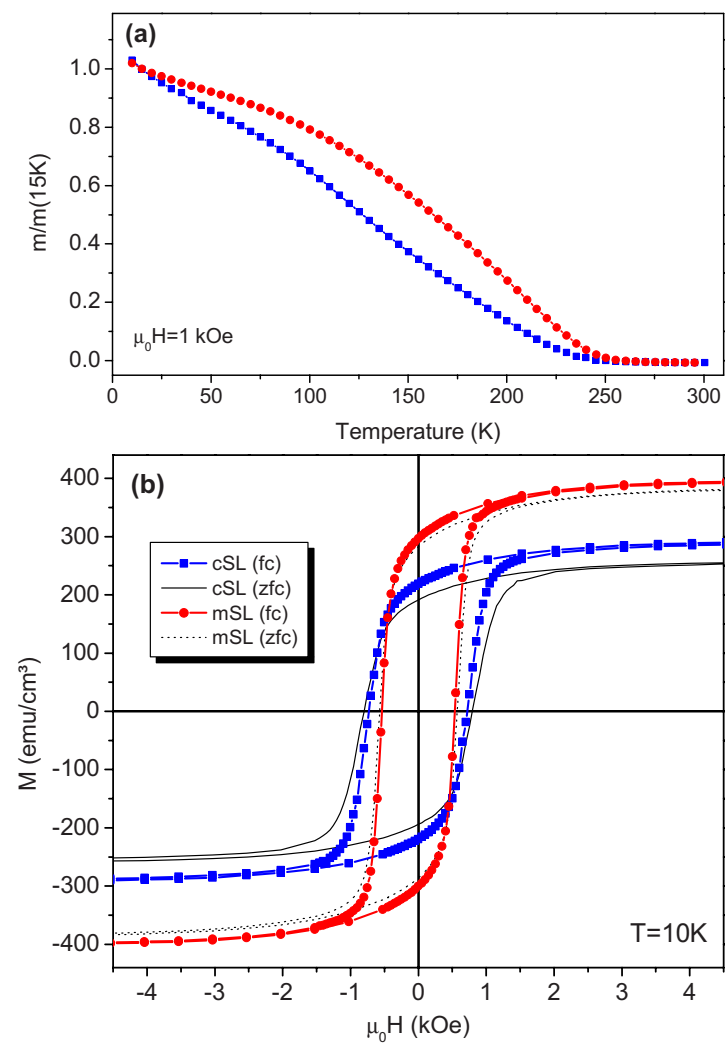

FIG. 4. (Color online) (a) Normalized magnetization as a function of temperature of the cSL (blue squares) and mSL (red circles). (b) Magnetic hysteresis at $T=10 \mathrm{~K}$ of cSL (blue squares) and $\mathrm{mSL}$ (red circles) after FC at $\mu_{0} H=5 \mathrm{~T}$ and cSL (solid line) and mSL (dotted line) after ZFC.

FC and ZFC condition. Two features in $K_{3 \omega}(T)$ are distinguishable: at $T=100 \mathrm{~K}$ and $T=255 \mathrm{~K}$ there exist kinks. Below $T=100 \mathrm{~K}$ the nonlinearity is very large, $K_{3 \omega} \approx-20 \mathrm{~dB}$,

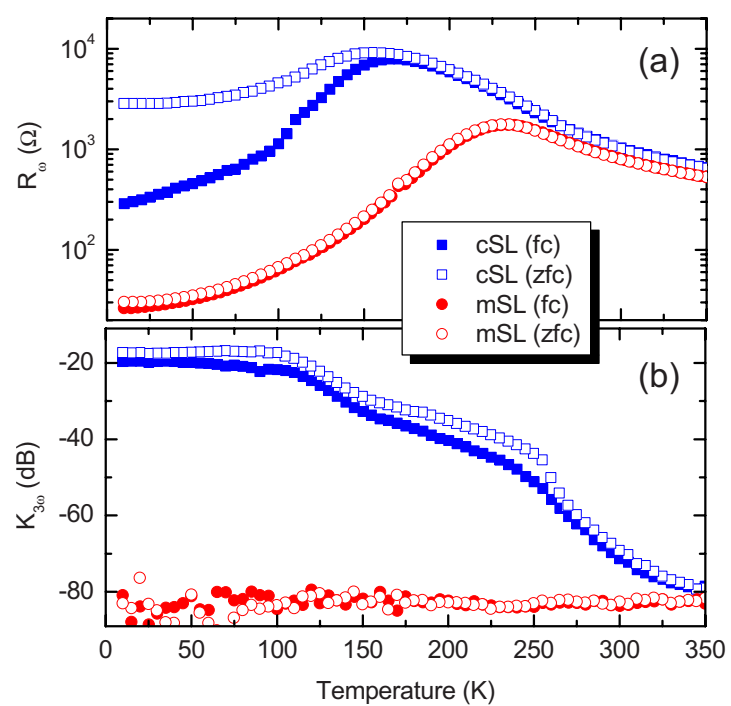

FIG. 5. (Color online) Transport properties of the cSL (blue squares) and mSL (red circles) as a function of temperature. (a) Linear ac resistance $R_{\omega}$ and (b) the nonlinear coefficient $K_{3 \omega}$ are shown for FC (closed symbols) and ZFC (open symbols) measurements. 
and nearly constant. For the mSL a very small nonlinear signal $\left(K_{3 \omega}<-80 \mathrm{~dB}\right)$ was measured in the whole temperature range, both for $\mathrm{FC}$ and $\mathrm{ZFC}$ condition.

In the case of manganite-titanate interfaces, the concept of interface doping leads to an overall increase in the Mn valence, which would be equal to an increase in doping to $x$ $=x_{\text {nominal }}+x_{\text {interface }}$. Recent theoretical and experimental studies show that the length scale on which charge transfer takes place is rather small, $l_{c t} \approx 1 \mathrm{~nm},{ }^{28,29}$ and therefore $x$ is increased only in the vicinity of the interfaces. By adding LMO layers we decrease $x_{\text {nominal }}$ in this region. The overall doping level at the interfaces of the $\mathrm{mSL}$ is smaller compared to that at the cSL interfaces. In this sense, the interface regions bring about different electronic constraints to the LCMO layers in the SLs. Because of the strong electronic correlations these electronic constraints have tremendous impact on the structural properties of the LCMO layers.

First of all the enhanced saturation magnetization $M_{s}$ and reduced coercivity $H_{c}$ in $\mathrm{mSL}$, see Fig. 4(a), directly show that the interface modification strongly reduces the tendency toward AFM correlation. The difference in $M_{s}$ after FC/ZFC and the extreme difference between $T_{c}$ and $T_{\mathrm{MI}}$ for the cSL underlines a pronounced magnetic inhomogeneity of the cSL. Due to the modification magnetic inhomogeneity is reduced in the mSL, which can also be seen in a somewhat steeper magnetic transition [see Fig. 4(b)]. The difference of the magnetic homogeneity is also reflected by the electric transport behavior of the SLs. The FC/ZFC difference, observed only for the cSL [see Fig. 5(a)], can be attributed to an inhomogeneous electronic (phase separated) state, which is known from $\mathrm{La}_{1-x} \mathrm{Ca}_{x} \mathrm{MnO}_{3}$ with $0.4 \leq x \leq 0.5$.

Most interestingly, the different electric constraints of the SLs not only change the magnetic and transport properties but also the structure of the interface region and hence of the whole LCMO layers. For LCMO without oxygen deficiency, it was shown that crystalline structure, determined by the amount of $\mathrm{Mn}^{4+}$ ions, changes from $R \overline{3} c$ for $x \leq 0.4$ to Pnma for $x \geq 0.5$. $^{30}$ The right oxygenation can be expected in the case of metal-organic aerosol deposition technique, as films are grown at ambient oxygen pressure. The manganite of the $\mathrm{mSL}$ shows $R \overline{3} c$ symmetry, which has been observed for small amounts of $\mathrm{Mn}^{4+}$ ions, i.e., $x \leq 0.4$. The LCMO layers of the cSL show Pnma symmetry, which is found for $x$ $\geq 0.5$. So, most probably the interface region of cSL is in the CE-AFM state, as it is found for LCMO with $x \geq 0.5$. As we have shown previously ${ }^{21}$ the manganites with $R \overline{3} c$ structure (LSMO) show linear transport behavior, whereas for the Pnma structure nonlinearity has been observed in the transition region. Here we see another example of this rule: the $\operatorname{mSL}(R \overline{3} c)$ shows a very small $K_{3 \omega} \approx-80 \mathrm{~dB}$, whereas the cSL (Pnma) shows pronounced nonlinear transport properties, see Fig. 5(b). The extremely large nonlinear signal, $K_{3 \omega}>-20 \mathrm{~dB}$, at low temperatures on the other hand is in contrast to the results for single LCMO films. ${ }^{21}$ This can be attributed to the high density of interfaces of the cSL compared to a single LCMO film. The interfaces act like nucleation centers, at which the $\mathrm{CP}$ can accumulate and therefore the amount of $\mathrm{CP}$ is enhanced in cSL. If one assumes the $\mathrm{CP}$ to be the building blocks of the $\mathrm{CE}$ phase, it is clear that the interface region is unstable against the antiferromagnetic $\mathrm{CE}$ correlations. ${ }^{19}$ For the mSL the number of $\mathrm{CP}$ is reduced so that $K_{3 \omega}$ is very small. Furthermore the Pnma symmetry, in contrast to the homogeneous $R \overline{3} c$ symmetry, allows for local elastic deformation. Phase separation into FM and CE-AFM, like it has been discussed for LCMO with $0.4 \leq x \leq 0.5,{ }^{8}$ can lead to the inhomogeneous magnetic and electric state of the cSL.

In summary we have shown that engineering of the doping profile in LCMO-BTO superlattices has massive influences on the magnetotransport properties. Only two monolayers of LMO at each interface lead to an enhancement of the saturation magnetization. Remarkable fact is that due to the interface modification also the crystal structure of the manganite layers changes. We discussed, that in conventional LCMO/BTO superlattices the ground state of the interfacial LCMO layers is likely CE-AFM, supporting theoretical calculations. ${ }^{19}$ This phase is responsible for exceptional nonlinear $(3 \omega)$ transport as well as for ZFC-FC splitting of the linear resistance discussed within correlated polaron model and phase-separation scenario.

This work was supported by DFG via SFB 602, TPA2.

\footnotetext{
${ }^{1}$ A. Ohtomo and H. Y. Hwang, Nature (London) 427, 423 (2004).

${ }^{2}$ S. Thiel et al., Science 313, 1942 (2006).

${ }^{3}$ E. Dagotto, Phys. 2, 12 (2009).

${ }^{4}$ N. Nakagawa et al., Nature Mater. 5, 204 (2006).

${ }^{5}$ G. M. Müller et al., Nature Mater. 8, 56 (2009).

${ }^{6}$ B. Nadgorny et al., Phys. Rev. B 63, 184433 (2001).

${ }^{7}$ P. Schiffer et al., Phys. Rev. Lett. 75, 3336 (1995).

${ }^{8}$ J. van den Brink et al., Phys. Rev. Lett. 83, 5118 (1999).

${ }^{9}$ D. I. Khomskii, Int. J. Mod. Phys. B 15, 2665 (2001).

${ }^{10}$ G.-m. Zhao et al., Phys. Rev. Lett. 84, 6086 (2000).

${ }^{11}$ A. S. Alexandrov and A. M. Bratkovsky, Phys. Rev. Lett. 82, 141 (1999).

${ }^{12}$ C. P. Adams et al., Phys. Rev. Lett. 85, 3954 (2000).

${ }^{13}$ C. S. Nelson et al., Phys. Rev. B 64, 174405 (2001).

${ }^{14}$ M. Bibes et al., Phys. Rev. B 66, 134416 (2002).

${ }^{15}$ M. Huijben et al., Phys. Rev. B 78, 094413 (2008).
}

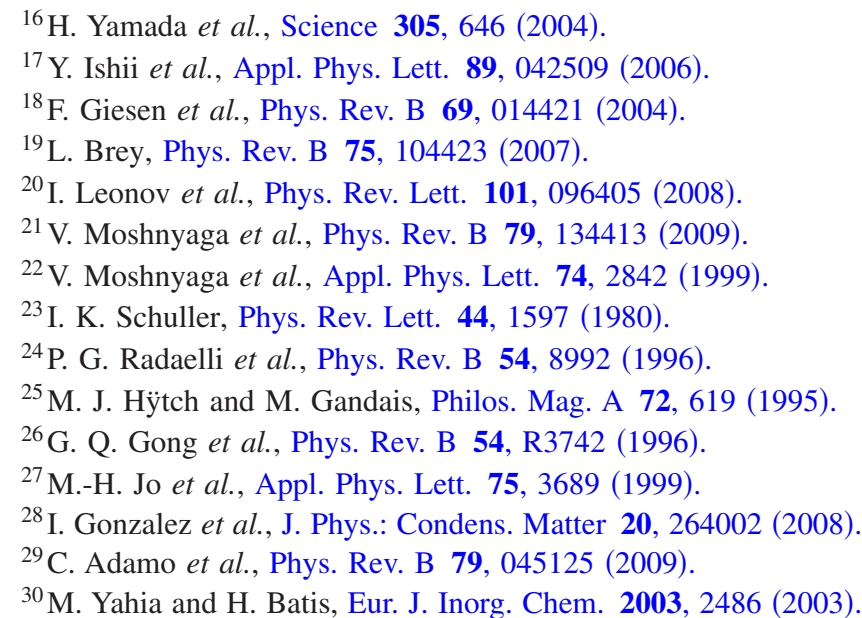

